\title{
Lipohémarthrose du genou : signe indirect de fracture articulaire
}

\section{Lipohemarthrosis of the Knee Joint as an Indirect Sign of Intra-articular Fracture}

\author{
P. Le Borgne $\cdot$ A. Perriguey $\cdot$ P. Kauffmann
}

Reçu le 18 avril 2014; accepté le 18 juillet 2014

(C) SFMU et Lavoisier SAS 2014

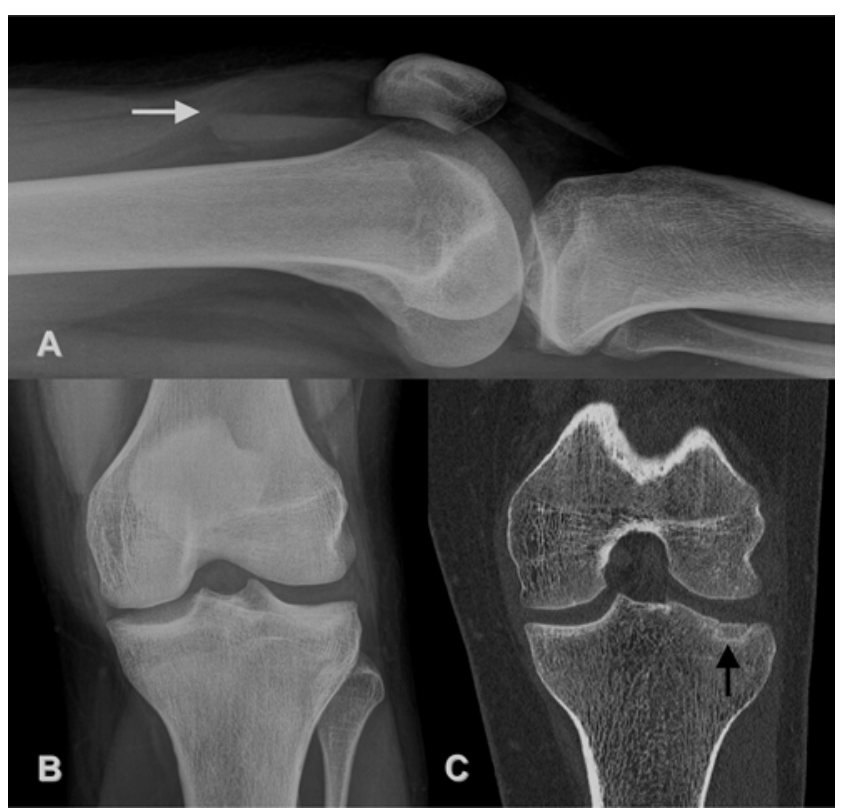

Fig. 1 A : Radiographie standard genou gauche, profil. Flèche blanche : niveau graissoliquidien supra-patellaire.

B : Radiographie standard genou gauche, face, normale.

$\mathrm{C}$ : Scanner genou gauche, face. Flèche noire : fracture-enfoncement du plateau tibial latéral

Un homme de 24 ans s'est présenté aux urgences pour douleur du genou gauche suite à un accident de la voie publique à faible cinétique en valgus forcé. L'examen clinique initial retrouve une franche impotence fonctionnelle et un épanche- ment articulaire avec comblement du cul de sac sousquadricipital gauche. La flexion du genou est limitée à $60^{\circ}$. Le reste de l'examen ne retrouve aucune autre anomalie. La radiographie de face apparaît normale. Le profil (Fig. 1) a montré un niveau horizontal graisso-liquidien ainsi qu'une rupture de la ligne convexe du plateau tibial externe. Un scanner a confirmé une fracture-enfoncement du plateau tibial latéral gauche peu évident initialement sur les radiographies. La lipohémarthrose correspond à une accumulation de graisse (provenant de la moelle osseuse) dans un épanchement articulaire hémorragique. Elle se forme habituellement dans les trois heures suivant le traumatisme. La description princeps de ce signe date de 1929 [1]. La sensibilité de ce signe est faible mais la spécificité est excellente [2]. La présence d'une lipohémarthrose en radiographie standard constitue une « lésion sentinelle » en traumatologie, elle suggère fortement une fracture articulaire. Son absence n'élimine en aucun cas une lésion fracturaire. Le traitement fut orthopédique : immobilisation par un plâtre cruropédieux en extension puis mobilisation progressive et reprise de l'appui à partir du deuxième mois.

\section{Références}

1. Kling DH (1929) Fat in traumatic effusions of the knee joint. Am J Surg 6:71-4

2. Lee JH, Weissman BN, Nikpoor N, et al (1989) Lipohemarthrosis of the knee: A review of recent experiences. Radiology 173: $189-91$

P. Le Borgne $(\bowtie) \cdot$ A. Perriguey $\cdot$ P. Kauffmann

Service d'accueil des urgences, CHU de Strasbourg,

hôpital de Hautepierre, 1, avenue Molière,

F-67098 Strasbourg, France

e-mail : pierrick_med@yahoo.fr 